\title{
Trabajo, ciudadanía social y riesgo biográfico en regiones periféricas
}

\author{
Juan Monreal \\ Andrés Pedreño \\ Universidad de Murcia. Facultad de Economía y Empresa \\ Departamento de Sociología y Política Social \\ Campus Universitario. 30100 Espinardo (Murcia) \\ jmonreal@fcu.um.es \\ andrespe@fcu.um.es
}

\section{Resumen}

El objetivo de este artículo es introducir la dimensión territorial en el análisis de la política social. De esta forma se observa un proceso de diferenciación del espacio europeo, en el cual una serie de regiones periféricas experimentan un proceso de extensión de categorías sociales vulnerables, que debilitan la ciudadanía social. La lógica social característica de estas regiones ha sido caracterizada como "regiones vulnerables», lo cual legitima un punto de vista territorial como el que aquí se propone. Nuestro punto de partida es un marco teórico que contempla los efectos de la reestructuración del trabajo sobre los derechos de la ciudadanía social, al tiempo que constata las líneas de incorporación de lo político en la esfera productiva.

Palabras clave: teoría de la ciudadanía, vulnerabilidad social y regiones, sociedad del riesgo.

\section{Resum. Treball, ciutadania social i risc biogràfic en regions periferiques}

L'objectiu d'aquest article és introduir la dimensió territorial en l'anàlisi de la política social. D'aquesta manera s'observa un procés de diferenciació de l'espai europeu, en el qual una sèrie de regions periferiques experimenten un procés d'extensió de categories socials vulnerables, que afebleixen la ciutadania social. La lògica social característica d'aquestes regions ha estat caracteritzada com a «regions vulnerables», la qual cosa legitima un punt de vista territorial com el que aquí es proposa. El nostre punt de partida és un marc teòric que contempla els efectes de la reestructuració del treball sobre els drets de la ciutadania social, al mateix temps que constata les línies d'incorporació d'allò polític en l'esfera productiva.

Paraules clau: teoria de la ciutadania de Marshall, vulnerabilitat social i regions, societat del risc.

\section{Abstract. Work, social citizenship and biographical risk in peripheral regions}

The objective of this article is to introduce the territorial dimension in the analysis of social policy. A process of diferenciacion of the European space is verified, in which a group of peripheral regions experiences a process of extension of vulnerable social categories that weaken social citizenship. The social logic of these regions has been characterized as «vulnerable regions». It legitimates the territorial perspective as we propose. Our starting point is a theoretical framework that considers the effects of current work restructuring regard- 
ing the rights of social citizenship. At the same it verifies the lines of incorporation of the political thing to the productive sphere.

Key words: citizenship's theory, social vulnerability and regions, risk society.

\section{Sumario}

1. Introducción 4. Experiencias laborales del riesgo:

2. La teoría de la ciudadanía de T.H. Marshall

3. De la seguridad social a la sociedad del riesgo indicios empíricos en la región de Murcia

5. Conclusiones: la producción de biografías sociales precarizadas

Bibliografía

\section{Introducción}

En el final del siglo XX hemos asistido a profundas transformaciones en las formas de vida y de trabajo, que se agudizarán en este siglo XXI. Estos cambios presentan carácter globalizador, al tener lugar prácticamente en cualquier espacio, independientemente de la extensión e intensidad de los mismos (Monreal, 1999).

Sin embargo, en Europa estos cambios se están acusando especial y básicamente en tres frentes de vital importancia. En primer lugar, en el mundo del trabajo y del empleo, debido, sobre todo, a unas formas globales de competencia y al advenimiento de la sociedad informacional. En segundo lugar, en las relaciones sociales. Éstas están cambiando, como resultado del alto nivel de complejidad que presenta hoy lo social, pero debido también a un avance en la emancipación y la diferenciación de las estructuras familiares. Y, por último, se están produciendo una serie de cambios sociodemográficos importantes, explicables desde el envejecimiento de la población y el creciente peso de la inmigración.

Europa, ante estos cambios y transformaciones, debe ir encontrando respuestas a las nuevas situaciones a través de sus políticas generales, tanto económicas como sociales, de modo que el ciudadano europeo no se sienta golpeado ni marginado por las nuevas realidades emergentes, sino que, al contrario, perciba cómo la Unión Europea es capaz de aplicar políticas y estrategias adecuadas que proporcionen las mejores condiciones posibles de vida, apostando decididamente por políticas que generen calidad social (Beck, Maesen y Walker, 1997).

La idea de una "civilización única» está presente en la misma constitución de las sociedades industriales occidentales. El desarrollo histórico de la tesis de progreso de la modernidad plantea que las contradicciones derivadas de la universalización de la producción (por ejemplo, el conflicto capital-trabajo) requieren instituciones de mediación (que cristalizarán en el Estado social de derecho), consumando definitivamente una "civilización común» en torno al ideal de progreso (Campillo, 1985). Esta conciliación entre los contrarios en el espacio social, se realizará sobre la base de la compatibilidad entre igualdad de estatus y desigualdad económica (Marshall, 1998/1950). 
Esta perspectiva, sin embargo, subvalora la naturaleza sociopolítica de nuestras sociedades avanzadas (Hindess, 1993). Una serie de procesos presentes en la estructura social, tales como la presencia de bolsas importantes de «subproletariado» en nuestras sociedades, con esa experiencia vital de «falta de porvenir» (Bourdieu, 1999a: 309) propia de esas situaciones sociales, o la persistencia de las discriminaciones de género y etnia, cuestionan la idea de una "civilización única», desde el momento que tales situaciones implican una considerable dificultad material de ejercicio pleno de sus derechos de ciudadanía.

Igual consideración cabe hacer de la idea de una Europa comunitaria o de una sociedad europea de la información, donde "las nuevas potencialidades ofrecidas a las personas exigen de todos un esfuerzo de adaptación, en particular para construir por sí mismos su propia cualificación» (Comisión Europea, 1995). Obsérvese en este texto el efecto de integración que busca el uso del término todos.

Creemos que el ideal de una "civilización única» tomó forma en la distinción, establecida durante el proceso de modernización propio de la sociedad industrial, entre un sistema político-administrativo, en el que rigen los principios de ciudadanía, y un sistema económico-técnico, en el que predominan los principios de productividad y competitividad (Pries, 1991). Durante cincuenta años los derechos de ciudadanía crecieron de forma continua y exponencial, hasta tal punto de dotar de una verdadera dimensión social al proceso económico de compra y uso de fuerza de trabajo. Precisamente la «edad de oro» del capitalismo industrial estuvo fundada en un pacto social de contrapartidas recíprocas entre capital y trabajo (Boyer, 1986), donde la expansión de la ciudadanía social y sus efectos de bienestar actuó de contrapeso a la conversión del trabajo en "apéndice de la máquina». Seguridad social y gran industria definían respectivamente y sincrónicamente al ciudadano y al obrero. Las políticas sociales se configuraron como seguridad social, y por tanto, la incertidumbre vital inherente a la lógica social de la gran industria se convirtió en objetivo a amortiguar.

Podemos leer la teoría de la ciudadanía de T.H. Marshall como un producto resultante de separar la esfera de la producción y la esfera de la ciudadanía. En el mundo de la producción, los individuos están posicionados desigualmente. En el mundo de la ciudadanía, los individuos están integrados en una misma «civilización» gracias al reconocimiento de unos derechos y deberes.

Hemos de reconsiderar críticamente esa perspectiva. Y, para ello, se requiere un enfoque que busque la interacción entre el sistema técnico-económico y el sistema político-democrático. El crecimiento del subproletariado o el uso de las desigualdades de género y etnia en el proceso de trabajo (Thomas, 1985; Pedreño, 2000), tienen que ver con procesos de politización en la esfera técnico-económica, al tiempo que los cambios que se están dando en el interior de la producción tienen efectos sobre el sistema político-democrático. Desde los años setenta una profunda reestructuración del trabajo hace emerger nuevas realidades sociolaborales que transforman las codificaciones fordistas entre trabajo y sociedad. En el ámbito del trabajo, la informalización y la desregulación 
del trabajo se extienden, horadando los presupuestos de la ciudadanía social. En el ámbito de la sociedad, en el modelo de estratificación social competitiva que caracteriza a la actual estructura social (Ortí, 1992), el riesgo y la incertidumbre se consideran factores positivos de estímulo a la libre competencia. El resultado de este conjunto de cambios es cada vez una mayor fractura entre trabajo y ciudadanía social. El derecho del trabajo sufre un retroceso que congela el crecimiento progresivo experimentado durante más de sesenta años. La seguridad social deja paso a la sociedad del riesgo.

La problemática que planteamos en este artículo incorpora la dimensión territorial de las desigualdades sociales. De este modo se constata un proceso de diferenciación del espacio europeo (Monreal, 1986), en el que una serie de regiones periféricas experimentan un proceso de extensión de categorías laborales vulnerables que debilitan a la ciudadanía social. La lógica social propia de estas regiones ha de tenerse muy presente para evaluar esa "civilización común» que se instituye como sociedad europea de la información.

\section{La teoría de la ciudadanía de T.H. Marshall}

T.H. Marshall propone, en su obra de 1950, una hipótesis sociológica que plantea la compatibilidad entre una igualdad básica fundamentada en los derechos formales de ciudadanía y las desigualdades de clase: «[...] hasta tal punto de que la propia ciudadanía se ha convertido, en ciertos aspectos, en el arquitecto de una desigualdad social legitimada» (Marshall, 1998/e.o. 1950: 22).

La idea de ciudadanía fue definida por Marshall como «aquel estatus que se concede a los miembros de pleno derecho de una comunidad. Sus beneficiarios son iguales en cuanto a los derechos y privilegios que implica. Aunque no existe un principio universal que determine cuáles son los derechos y las obligaciones, las sociedades donde la ciudadanía es una institución en desarrollo crean la imagen de una ciudadanía ideal que sirve para calcular el éxito y es objeto de las aspiraciones. Las conquistas que se producen en la dirección así trazada proporcionan una medida más acabada de la igualdad, un enriquecimiento del contenido de ese estatus y un aumento del número de los que disfrutan de él» (Marshall, op. cit.: 37).

Comprender el despliegue histórico de la idea de ciudadanía es el objetivo del estudio de Marshall. Para ello propone «una división de la ciudadanía en tres partes» (p. 22), que se corresponde con respectivos estadios de la historia del capitalismo. Así señalará un «elemento civil» (los derechos necesarios para la libertad individual), un "elemento político» (el derecho a participar en el ejercicio del poder político $)^{1}$ y, por último, un «elemento social» que «abarca todo el espectro, desde el derecho a la seguridad y a un mínimo bienestar eco-

1. El desarrollo de la negociación colectiva en el mundo del trabajo lo contempla dentro de la ciudadanía política: "[...] el sindicalismo ha creado un sistema secundario de ciudadanía industrial paralelo al sistema de ciudadanía política, y complementario de él» (Marshall, op. cit.: 49). 
nómico al de compartir plenamente la herencia social y vivir la vida de un ser civilizado conforme a los estándares predominantes en la sociedad» (Marshall, op. cit.: 23).

Es en el periodo posterior a la Segunda Guerra Mundial cuando se consolida en los países del capitalismo avanzado un sistema de ciudadanía plena, que Marshall no lo entiende como un medio de igualar las rentas, sino como un dispositivo por el cual minimizar los riesgos sociales que el mercado introduce en la vida social. Es precisamente este hecho lo substancial del problema planteado por Marshall. Los derechos de ciudadanía producen «un enriquecimiento general del contenido concreto de la vida civilizada, una reducción generalizada del riesgo y la inseguridad, una igualación a todos los niveles entre los menos y los más afortunados» (Marshall, op. cit.: 59). En definitiva, para Marshall la ciudadanía posibilita «una población cohesionada por una civilización única» (p. 75).

Sobre la teoría de ciudadanía de Marshall se han realizado numerosas críticas, destacando unos su "anglocentrismo» (Turner, 1993), y otros su perspectiva evolucionista del cambio social (Giddens, 1996). Sin embargo, solamente aquéllas que han centrado sus críticas en la noción de "civilización única» presentan un cuestionamiento del núcleo central de la teoría de Marshall, al desvelar la inconsistencia de una visión de las sociedades occidentales como si fueran "comunidades de ciudadanos» (Hindess, 1993). Por ejemplo, Bottomore (1998) resalta las desigualdades étnicas y de género existentes en nuestras sociedades, e incide en una cuestión que Marshall trata con ligereza, el hecho de «las limitaciones económicas y de clase que se oponen al ejercicio efectivo de los derechos formalmente establecidos» (Bottomore, 1998: 137). Para Hindess (1993), Marshall acierta en su insistencia sobre el significado del principio de ciudadanía en el discurso político contemporáneo, pero se equivoca en su tratamiento de la ciudadanía como un estatus que ha sido más o menos adecuadamente realizado. Y destaca dos de los puntos donde la teoría de Marshall es inadecuada: a) en su concepción liberal de la ciudadanía como una persona libre e independiente protegida por derechos, que le lleva a minusvalorar «la forma en que tienen consecuencias la influencia de las ideas políticas (y de otras)» (Hindess, 1993: 33), y b) en la ausencia de un análisis de las relaciones entre género y ciudadanía, que impide a la teoría de Marshall comprender que «la significativa importancia del principio de ciudadanía en el discurso político occidental no asegura que hombres y mujeres serán tratados igualitariamente» (p. 33).

\section{De la seguridad social a la sociedad del riesgo}

Tras la crisis económica de los años setenta, una serie de sucesivas reestructuraciones económicas y sociales extienden las situaciones de riesgo social. Beck ha teorizado el actual cambio social como la transformación de una sociedad industrial, basada en la producción social de riqueza, a una sociedad del riesgo, basada en la producción social de riesgos (Beck, 1998a). 
Para Beck la amplitud de la esfera del riesgo y de la incertidumbre es el rasgo más destacado del capitalismo tardío. Si en la sociedad industrial el eje de producción definía la distribución de los recursos entre capital y trabajo, lo que caracteriza a la sociedad del riesgo es una nueva forma de estratificación social generada por la producción y la distribución del riesgo. La crisis ecológica, el cuestionamiento del proyecto de capitalismo de bienestar y la globalización son las tendencias sociales básicas sobre las que se edifica la sociedad del riesgo. Los riesgos ambientales traspasan las naciones. El capital circula por flujos globales financieros imponiendo a los Estados nación progresivamente debilitados sus condiciones de rentabilidad. La globalización de lo social también erosiona cada vez más las viejas fronteras nacionales. En este mundo inestable, la multiplicación del riesgo impone una lógica propia de estructuración social.

Una de las ideas más destacables de Beck es su concepción de la sociedad del riesgo como una nueva politización de lo social. Esta «subpolítica» cristaliza en una serie de ámbitos diversos y en direcciones diferentes.

En primer lugar, se observa una (re)politización del empresariado. La crisis del capitalismo de bienestar y la globalización de la economía ha supuesto el retorno de una considerable cuota de poder político al empresariado. Una lectura empresarial de la realidad que enfatiza como males de la economía todas aquellas variables socioeconómicas que impiden el funcionamiento de la lógica pura del mercado, ha terminado imponiéndose.

$\mathrm{Su}$ poder se basa, como indica Beck (1998b: 169), en una «escenificación del riesgo", en donde toda una serie de discursos, que adoptan la forma linguística de la amenaza, se ponen en circulación para obligar al resto de actores sociales y políticos a adoptar las medidas necesarias para fijar las condiciones más ventajosas para la rentabilidad de las inversiones de capital, que son siempre aquéllas que garantizan la plena hegemonía del mercado. De esta forma, la globalización posibilita que «los empresarios, sobre todo los que se mueven a nivel planetario, puedan desempeñar un papel clave en la configuración no sólo de la economía, sino también de la sociedad en su conjunto, aun cuando sólo fuera por el poder que tienen para privar a la sociedad de sus recursos materiales (capital, impuestos, puestos de trabajo) (Beck, 1998b: 16).

Es este tipo de sociedad del riesgo lo que ha posibilitado que en todas partes asistamos a la introducción de medidas tales como la reducción del gasto público hasta cuestionar la estabilidad del Estado de bienestar, la creciente privatización de parcelas del ámbito colectivo, la flexibilidad del mercado de trabajo, etc. Del mismo modo, como la globalización conlleva, al mismo tiempo, un proceso de localización, los poderes regionales económicos y políticos han recogido los papeles de la "escenificación del riesgo" para moldear sus sociedades como "regiones vulnerables", en una estrategia competitiva por reducción de costes que busca atraer inversiones del capital global o asegurar la inserción de los sistemas productivos locales en el mercado global.

Esta «subpolítica» conforma nuevos espacios de riesgo, esto es, la introducción a gran escala de la inseguridad en el horizonte del asalariado ante la 
continua expansión de la precariedad laboral y el desempleo en un proceso de continua y permanente reconversión del tiempo laboral. Como ha escrito Bourdieu (1999b: 141) a propósito del proyecto neoliberal «el fundamento último de todo ese orden económico situado bajo la invocación de la libertad de los individuos es, en efecto, la violencia estructural del paro, la precariedad y el miedo que inspira la amenaza del despido: la condición del funcionamiento "armonioso" del modelo micro-económico individualista [...] reside, en último término, en un fenómeno de masas, la existencia del ejército de reserva de los parados».

De esta forma, también la sociedad del riesgo entraña una politización de la esfera del trabajo. En efecto, la cuestión de la exclusión social y, consiguientemente, el debilitamiento de la ciudadanía social, no cabe comprenderla separada de la esfera del trabajo. En efecto, las empresas y sus decisiones instrumentales generan problemas de exclusión social. En este sentido, Robert Castel ha propuesto la noción de vulnerabilidad social como proceso social que articula la crisis del trabajo, del empleo y de la política con las situaciones de pauperización y exclusión social: «sería mejor poner en relación lo que está ocurriendo en las situaciones de marginalidad extrema, de aislamiento social, de pobreza absoluta con la configuración de situaciones de vulnerabilidad, de precariedad que, con frecuencia, las preceden y alimentan» (Castel, 1995: 28).

La constitución de regiones vulnerables implica un enorme riesgo potencial de deterioro de la calidad social de estos espacios, si nos atenemos al concepto dinámico de vulnerabilidad social propuesto por Castel. Por ello, la compresión sociológica de estos procesos se torna objetivo prioritario en aquellas regiones donde los indicios de sujetos sociales frágiles (Valera y Álvarez-Uría, 1989) hacen pensar que la vulnerabilidad social se ha localizado en el mismo centro de su estructura social.

\section{Experiencias laborales del riesgo: indicios empíricos en la región de Murcia}

Desde hace unos años investigamos sobre las condiciones de trabajo y las experiencias de vida de las nuevas realidades obreras presentes en la región de Murcia (Monreal, 1991). Nos hemos interesado por plantearnos interrogantes como los que siguen: ¿cómo se manifiesta en las regiones meridionales la fractura entre trabajo y ciudadanía social?, ¿en qué está consistiendo la experiencia laboral de la incertidumbre y del riesgo en estas regiones? Lo que aquí presentamos son nuestros primeros datos, a espera de que la investigación nos permita seguir profundizando.

Ha llamado la atención el sociólogo italiano Arnaldo Bagnasco sobre la necesidad de afrontar la presente reestructuración de la estructura social de «una forma no genérica» (Bagnasco, 1997: 115), proponiendo, más bien, «encontrar figuras y relaciones sociales específicas, en zonas específicas de la economía» (p. 89). Atendiendo a esta advertencia metodológica, diremos que la base material de la región de Murcia es un capitalismo de pequeña y media- 
na empresa, mayormente rural, aunque no exclusivamente. No es aquí el lugar para desarrollar ni defender tal tesis. Nos conformamos con situarnos en ese punto de partida de cara a explorar algunas de las figuras obreras que aparecen en esa "zona específica de la economía», para extraer algunas reflexiones que nos permitan vislumbrar indicios de tendencias de una situación social que, cada vez más, define un espacio regional de vulnerabilidad social.

Una vez situados en la realidad concreta, proponemos centrarnos en cinco cuestiones relevantes de las relaciones de trabajo presentes en la región murciana: la centralidad del trabajador eventual en la estructura social; los cambios en las relaciones de trabajo introducidos por la implantación en la región de un potentísimo polo productivo en torno a la agricultura industrial; la progresiva feminización de sistemas productivos relevantes de la economía murciana; la presencia cada vez mayor de una fuerza de trabajo inmigrante, y los procesos de nomadismo laboral.

\section{La eventualidad del trabajo en el centro de la estructura social regional}

Los datos de la Encuesta de Población Activa (EPA, tercer trimestre de 1999) indican que la mitad de la población adulta murciana se define como «activa» (es decir, un $51,3 \%$ está ocupada o busca trabajo), de la cual un $13,9 \%$ se incluye dentro de la población desempleada. Todo ello nos advierte que un $44,2 \%$ de la población adulta está dentro del espacio de las posiciones sociales que disponen del medio que se considera más importante para acceder a la distribución de la riqueza regional. Si los datos estadísticos se analizan desde la perspectiva del género, se aprecian que son las mujeres las que menos posibilidades tienen de acceder a un trabajo remunerado (un 29\% frente a un $60 \%$ de los varones), las que sufren las mayores tasas de paro (un $22,1 \%$ frente al $9,2 \%$ de los varones), etc.

Sin embargo, la realidad dibujada por la estadística es solamente una aproximación a la realidad dibujada por la informalización del empleo y la economía sumergida, que aparece como opaca para las estadísticas oficiales. La presencia del empleo informal y sumergido (trabajos remunerados sin contrato ni cotización a la Seguridad Social) alcanza, según los estudios más recientes, las tasas más elevadas del conjunto del territorio español. Ya a mediados de los 80 una amplia encuesta del gobierno constataba tal afirmación, de manera que esta situación afectaba a casi un tercio de sus trabajadores $(32 \%)^{2}$.

2. La referencia es Secretaría General de Economía y Planificación (Ministerio de Economía) y CIS (1986). Un reciente trabajo realizado por la Universidad de Zaragoza, coordinado por el profesor Jose María Serrano, bajo el título de «Desigualdades Territoriales en la Economía Sumergida» (1999), ofrece los datos más actualizados hasta ahora en España sobre el peso de la economía sumergida. En dicho estudio la región de Murcia aparece con el mayor porcentaje de irregularidad laboral del conjunto de España, con un 30,3\%, seguido de Baleares con un 23,5\%, Galicia con un 22,3\%, etc. En cuanto al peso de la economía sumergida en el Producto Interior Bruto (PIB), la región de Murcia alcanza un 14,6\%. 
Estas pinceladas sobre el mercado de trabajo regional muestran que la enorme tasa de eventualidad laboral existente en la región (situada desde hace años, según la EPA, alrededor del $40 \%$ de la población ocupada) está haciendo de un auténtico puente entre la zona de integración social del empleo formal y la zona de vulnerabilidad del empleo sumergido. La eventualidad se ha instalado en el mismo centro de la estructura social, y prácticamente todas las categorías ocupacionales se ven afectadas por la misma. Y es precisamente este hecho el que precede y alimenta aquellos ámbitos del espacio social que concentran, de forma cada vez más ampliada, la población vulnerable y/o excluida.

A continuación se analiza un sistema productivo concreto, el de la agricultura industrial. Es un sector con una importancia central para la economía regional murciana, y al mismo tiempo refleja una situación «límite» de mercado de trabajo. Ello lo convierte en un escenario privilegiado donde detectar las realidades sociales que este artículo analiza.

\section{Reestructuración del trabajo en la agricultura: del jornalero al obrero de las factorías vegetales}

La economía murciana ha pasado de representar un estrecho $1,7 \%$ de la producción final agraria española en 1950 a contribuir con el 5\% en 1992 . La expansión espectacular del regadío, que casi se ha triplicado en estos años ${ }^{3}$, permite el asentamiento de un potentísimo polo productivo agrícola, principalmente en torno a la hortofruticultura en fresco, que explica el protagonismo agrario de Murcia en el contexto español. Alrededor del regadío ha crecido una importantísima industria alimentaria, que se ha convertido en un sector especialmente estratégico en la estructura económica regional, sobre todo por los efectos dominantes y dinámicos que genera sobre el resto de la actividad económica.

La globalización de la agricultura hortofrutícola en fresco implica una nueva división internacional del trabajo (Bonnano, 1994: 17-18), por la cual los centros de consumo y las cadenas de distribución se concentran en los países del capitalismo avanzado (USA, Europa y Japón), mientras que las unidades de producción tienden a implantarse en los países del sur. Esta misma división internacional del trabajo se proyecta sobre el mapa europeo. En efecto, a las zonas tradicionales de producción intensiva de productos hortofrutícolas o de flores, tales como el sur de Inglaterra o Holanda, se suman los nuevos espacios productivos del Mediterráneo, cuyo gran atractivo reside en las ventajas climáticas y, sobre todo, en la amplia disponibilidad de mano de obra abundante y a bajo precio.

De esta forma ha terminado consolidándose una nueva división intraeuropea del trabajo agrícola: es en los países centrales de la Unión Europea donde

3. La superficie de regadío pasa de 69.700 hectáreas en 1950, a las 191.600 hectáreas en 1992. 
se definen los nichos de consumo y los mercados de primera calidad del producto en fresco, así como el control de la comercialización por parte de las grandes cadenas de distribución, mientras que en los países mediterráneos se concentra la producción. Igualmente, es en las regiones del norte donde se dan los procesos de Investigación y Desarrollo (semillas, fitosanitarios, investigación genética, etc.), mientras que en el sur se manifiesta una dependencia tecnológica de la producción (Pedreño, 2000b).

La especialización regional de la producción agrícola está íntimamente ligada a la disponibilidad de un mercado de trabajo abundante, y cuyo coste de mano de obra es extremadamente bajo. Esta especialización productiva se muestra en su organización del trabajo altamente consumidora de energía humana. En efecto, la nueva agricultura conlleva un proceso de salarización importante sobre el total de la población activa agraria. Las principales causas explicativas de este fenómeno responden a tres procesos: 1) la concentración/centralización de capital en unidades productivas relativamente grandes, 2) la necesidad de una cuantiosa mano de obra requerida por los cultivos intensivos, 3) la expansión de la fase del proceso de manipulado y «confección» del producto agrícola para incorporarle valor añadido al mismo.

La estructura ocupacional de la agricultura murciana está definida por seis dimensiones, tal y como viene avalado por los datos estadísticos de apoyo:

\section{Según modo de vinculación a la actividad}

La estructura ocupacional de la agricultura murciana presenta como rasgo definitorio la amplia base de relación salarial sobre la que se sustenta. Puede verse en la tabla 1 que la tasa de salarización representa un total del 71,38\%. Es decir, según la ratio, tres de cada cuatro activos agrícolas son asalariados 4 .

Tabla 1. Tasa de salarización por sexo en el sector agrario de la región de Murcia en 1991.

\begin{tabular}{lllc}
\hline & Total & Varones & Mujeres \\
\hline Tasa de asalarización & $70,9 \%$ & $67,8 \%$ & $82,6 \%$ \\
Población asalariada & 29.418 & 22.145 & 7.273 \\
Total de población ocupada & 41.458 & 32.656 & 8.802 \\
\hline
\end{tabular}

Fuente: elaboración propia a partir del censo de población de 1991, INE.

4. Etxezarreta (1994) confirma esta tendencia de formación de una agricultura salarial, a nivel de la agricultura española: «Actualmente en todas las zonas agrarias se está expandiendo una agricultura de tipo empresarial que recurre cada vez con más frecuencia y amplitud al trabajo asalariado, tanto fijo como, y sobre todo, eventual» (p. 124). «El trabajo asalariado ha aumentado desde el $28,6 \%$ en 1982 al $31,3 \%$ en 1987 , o desde el $36,5 \%$ en el mismo año base al 38,9\% en 1990, según la fuente que se utilice» (p. 135). 
Tabla 2. Evolución de la población ocupada del sector agrario según la situación profesional de la región de Murcia entre 1981 y 1991.

\begin{tabular}{lrrr}
\hline & & & \multicolumn{2}{c}{ Tasa media de crecimiento } \\
\hline Empresarios con asalariados & \multicolumn{1}{c}{ 1981 } & \multicolumn{1}{c}{$\mathbf{1 9 9 1}$} & \multicolumn{1}{c}{$\mathbf{1 9 8 1}$-1991 } \\
Empresarios sin asalariados & 10.755 & 1.552 & $128,9 \%$ \\
Miembros de cooperativas & 494 & 174 & $-12,8 \%$ \\
Asalariados fijos & 5.955 & 5.322 & $-64,8 \%$ \\
Asalariados eventuales & 29.586 & 24.096 & $-11,9 \%$ \\
Ayudas familiares & 1.904 & 843 & $-18,6 \%$ \\
Otra situación & 197 & 93 & $-55,7 \%$ \\
Total & 49.567 & 41.458 & $-52,8 \%$ \\
\hline
\end{tabular}

Fuente: elaboración propia a partir de los censos de población de 1981 y 1991, INE.

\section{Según situación profesional}

La preeminencia de la relación salarial como relación social básica de la agricultura murciana también se refleja en los datos de la tabla 2. Los datos de esta tabla muestran la importante polarización entre propietarios y asalariados. Nótese que el único grupo que crece en volumen entre 1981 y 1991 son los «empresarios con asalariados». La agricultura familiar pierde importancia progresivamente, hecho que motiva que las «ayudas familiares» se reduzcan a la mitad en el mismo periodo.

\section{Según modalidad de contratación}

La eventualidad continúa siendo el rasgo básico de la relación salarial agrícola. Alrededor del $60 \%$ de los ocupados agrarios son eventuales (tabla 2).

El marco institucional continúa reproduciendo el jornalerismo eventual como relación laboral básica, en una agricultura que ha consolidado definitivamente las relaciones sociales de producción capitalistas. Las condiciones concretas de débil vertebración de la estructura social por el modelo de industrialización, permitió la persistencia de un excedente de población rural, obligado a emigrar o empujado a circular en el empleo estacional, eventual y/o informal.

Posteriormente, tras el impacto de la crisis de 1973, las políticas de flexibilidad laboral, la dura competencia internacional de los mercados y el retorno de la emigración, suponen el asentamiento de nuevas condiciones para traer nuevas formas a la tradicional eventualidad jornalera. Aunque, al mismo tiempo, ha de destacarse cierta estabilidad en la evolución de los asalariados fijos y una (muy) leve tendencia a la disminución de los trabajadores eventuales.

\section{Según la división del trabajo por género}

En cuanto al género (tabla 3), entre 1981 y 1991 los asalariados agrícolas varones pierden la tercera parte de sus efectivos, al tiempo que las mujeres crecen más del doble. 
Tabla 3. Evolución de los asalariados agrícolas en la región de Murcia entre 1981 y 1991 por sexo.

\begin{tabular}{lcr}
\hline & Varones & Mujeres \\
\hline 1981 & 33.392 & 3.390 \\
1991 & 22.284 & 7.308 \\
Tasa media de crecimiento 1981-1991 & $-33,3 \%$ & $122,1 \%$ \\
\hline
\end{tabular}

Fuente: elaboración propia a partir de los censos de población de 1981 y 1991, INE.

En el proceso de trabajo agroindustrial y en la agricultura de los regadíos intensivos del litoral murciano, donde mayormente se concentra la demanda de mano de obra asalariada, la importancia de la actividad femenina es considerable 5 .

\section{Según la división del trabajo por etnia}

Progresivamente, la relación salarial en la agricultura murciana va adquiriendo una componente étnica cada vez más importante. Además de la etnia autóctona, «invisible» y marginada constituida por la población gitana, los trabajadores inmigrantes procedentes del Tercer Mundo - la mayor parte de procedencia marroquí- están realizando las tareas del campo, lo que da lugar a nuevos fenómenos sociológicos y económicos.

Secularmente un número indeterminado de población gitana, muchas veces de carácter nómada e itinerante, ha trabajado de jornalero en el campo como forma de obtención de unas rentas que le permitan subsistir en su — también secular-condición de marginalidad social.

Desde mediados de los ochenta, un contingente cada vez mayor de marroquíes ha ido insertándose en un mercado de trabajo con crecientes dificultades de mano de obra local y con tendencia al control sindical. Ambas dinámicas se han solventado para la empresa agrícola por la disponibilidad de esta mano de obra abundante, barata y disciplinada. También en los últimos años, aunque en menor medida que la población marroquí, ha ido asentándose en los campos murcianos un número reducido, pero creciente, de trabajadores argelinos, senegaleses, ecuatorianos, oriundos de países del este de Europa, etc.

5. El análisis de un municipio concreto con un enorme peso de la agricultura industrial, el caso del mercado de trabajo agrícola del municipio costero de Mazarrón (con una población de hecho de 15.250 personas), da cuenta de lo marcado de esta tendencia. Este municipio, junto con Águilas, también municipio costero, concentra las empresas más importantes de la producción de tomate de invernadero.

En efecto, el análisis destaca la mayor participación de la mujer en el mercado de trabajo en Mazarrón que en la totalidad de la región, con una tasa de actividad mucho más alta, tanto para 1986 (un 29,65\% frente al 21\%) como para 1991 (un 38,26\% frente al 29,7\%). Igualmente, la tasa de ocupación es mayor para la mujer en Mazarrón que en la región: en 1986 un 89,8\% en Mazarrón frente al 73,28 de la región; en 1991 un 82,13 frente a un $75,22 \%$. La tasa de paro femenina, por tanto, es menor en Mazarrón que en la región. 


\section{Según su procedencia geográfica}

El fenómeno de mayor relevancia a nivel de movilidad laboral lo constituye una cantidad indeterminada, pero muy importante cuantitativamente (podrían ser varios miles), de jornaleros andaluces que se desplazan estacionalmente todos los años para trabajar en la agricultura murciana. Las peonadas trabajadas en la empresa agraria murciana se contabilizan a la hora de cobrar el subsidio de desempleo agrario existente en Andalucía y Extremadura. También se da una movilidad geográfica intrarregional.

Concluyendo, la complejidad organizacional requerida por el potentísimo polo productivo agrícola murciano continúa, de forma paradójica, reproduciendo las viejas prácticas de eventualidad laboral, y ello es viable a través de la movilización de categorías sociales altamente vulnerables en el interior del proceso de trabajo (mujeres, inmigrantes y obreros nómadas). En definitiva, el caso de la agricultura industrial murciana recoge con rotunda claridad una serie de tendencias en su mercado de trabajo, que, aún en su manifestación más extrema, sintetizan los rasgos más sobresalientes que están definiendo un importante espacio social de vulnerabilidad dentro de la estructura regional del mercado de trabajo: eventualidad e informalización del empleo, feminización y etnificación del mercado de trabajo, polarización entre los empleos de las nuevas clases funcionales o de servicios que crecen a la par que el subempleo flexible, intensa movilidad territorial del trabajo (Pedreño, 1999a y 2000a).

\section{Mujeres en la economía informal ${ }^{6}$}

En la región murciana son muchas las mujeres que están incorporándose al creciente escenario de la economía informal que caracteriza a muchos de los procesos productivos de este territorio: agricultura industrial, industria del textil y del calzado, fábricas de conserva y de manipulación del producto agrícola en fresco, servicios y hostelería, etc. En los diversos estudios que hemos realizado, se constata una estructura de empleo claramente delimitada y segmentada según patrones de género.

Históricamente las tareas estacionales de numerosas formas de industria rural han estado ligadas al trabajo femenino. Este mecanismo de segmentación del trabajo, según género, ha continuado funcionando en la actualidad. En el apartado anterior sobre la agricultura industrial se ha observado el creciente protagonismo del empleo femenino, hasta tal punto que numerosas localidades de la región se han convertido en verdaderas áreas de especialización en el suministro de trabajo femenino. También hemos constatado la enorme presencia de la mujer en el trabajo sumergido, y su enorme significación para explicar la inmersión en la economía informal del sector industrial de la conserva, tradicionalmente de gran peso económico en la zona y en el conjunto regional (Pedreño, 1998).

6. En los tres epígrafes que siguen se presentan algunas de las biografías que estas realidades laborales están generando. 
De estas forma, las mujeres, en su busca de una mayor libertad individual, al incorporarse a este tipo de mercados de trabajo flexibles e informales, se encuentran con que el fraude generalizado a la seguridad social de las empresas con las que trabajan, les niega una historia laboral, con las consiguientes repercusiones a la hora de acceder a las prestaciones estatales. Ello se proyecta en una vida social coartada por el "no tener días» cotizados, como ellas mismas lo refieren, y la inestabilidad laboral. La vivencia laboral de la incertidumbre y el riesgo de estas mujeres obreras constituye un discurso agónico, donde la intensificación del trabajo, en un contexto de informalización del empleo, unifica al tiempo penosas condiciones de trabajo en materia de salud e higiene, escasas posibilidades de acción colectiva obrera por la ausencia de una mínima democracia laboral y crecientes dificultades para una vida social plena. Desde su experiencia de trabajo, el acceso a una ciudadanía social es un camino tortuoso e imposible.

El trabajo a domicilio en la industrial textil o en la industria del calzado (por citar dos casos típicos, pero habría otros: limpieza de productos hortícolas, fábricas de cascos de motos o de juguetes, etc.) es otra experiencia laboral de numerosas mujeres.

Lo que venimos observando en la región murciana alrededor de la categoría de género, es un coherente proceso de construcción social de una mano de obra al mismo tiempo estable y flexible. Este proceso está adquiriendo una sólida base material, con la articulación entre sí de los mercados de trabajo propiamente femeninos para reproducir y alimentar el doble rol de la mujer, asalariada y ayuda familiar.

En efecto, las prácticas de gestión empresarial de la mano de obra vigentes en la agricultura industrial son difícilmente compatibles con las tareas domésticas del cuidado de los hijos, por los largos horarios de trabajo y la desregulación existente. Ello incentiva a las mujeres en esa situación a abandonar el trabajo y permanecer al cuidado de las tareas domésticas. Pero, en realidad, lo que abandonan es el trabajo asalariado fuera del domicilio, pues es bastante frecuente que estas mujeres, cuando tienen hijos, opten por el trabajo a domicilio, cosiendo zapatos, confeccionando jerseys, etc. Conforme la edad de las mujeres trabajadoras se muestra inadecuada para el alto consumo físico que la agricultura industrial exige, ellas mismas abandonan o las propias empresas las sustituyen por mano de obra más joven. Llegado ese momento, la asistencia doméstica es el camino que eligen muchas de estas mujeres.

Esta movilidad interocupacional por diversos mercados de subempleo se funda en un escenario de riesgo caracterizado por la ausencia o debilidad de derechos de ciudadanía. Estas mujeres construyen biografías escritas sobre la constante entrada y salida del mercado de trabajo y la búsqueda de compatibilidad entre empleo y familia. La ausencia o debilidad de los sistemas institucionales de regulación empuja constantemente a estas figuras obreras a asumir un alto riesgo en sus biografías laborales, que las mantiene permanentemente en un territorio social de vulnerabilidad y exclusión. 


\section{Inmigrantes del Tercer Mundo: laboralmente segmentados, territorialmente desconectados}

Se calcula en unos 16.000-20.000 los inmigrantes extracomunitarios existentes en la región de Murcia, fundamentalmente marroquíes (un 86,1\% del total de inmigrantes), de los cuales aproximadamente entre el $40-50 \%$ podrían estar en condiciones de irregularidad. Junto a este problema aparece el de la temporalidad de los permisos de trabajo, sobre todo de los que tienen menos de un año de duración, que son la mayoría, dado el tipo de mercado de trabajo al que acceden los inmigrantes.

La legislación de extranjería sitúa a los inmigrantes regularizados en un universo de "ciudadanía de excepción», mientras que los irregulares son directamente "no-ciudadanos». Además, la especialización de ambos tipos de inmigrantes en el subempleo y/o en la economía informal degrada aún más sus débiles derechos de ciudadanía.

La vulnerabilidad social de los inmigrantes se deriva de su condición de ser expropiado de su condición de ciudadanía. Así lo han expresado numerosos trabajadores inmigrantes en las entrevistas realizadas en nuestras investigaciones: «[...] nosotros siempre lo pensamos que falta una cosa, cuando, si pensamos qué cosa falta, lo encontramos, que no estamos en nuestro país. Nosotros lo pensamos siempre que falta una cosa, faltamos algo en nuestro cuerpo, pero cuando lo pensamos, encontramos, estamos aquí, un inmigrante, y en otro país, ya no estamos en nuestro país y...» (trabajador marroquí).

Los dispositivos institucionales y la discriminación social del inmigrante están funcionando como fuerzas constitutivas de un mercado de trabajo de mano de obra volátil y flexible, que externaliza la reproducción social del trabajador inmigrante a su comunidad de origen. De esta forma se constituye una población en permanente movimiento, auténticos nómadas laborales, a los que se contrata en función de las necesidades productivas.

Sobre la desconexión institucionalizada de la población inmigrante, tanto del sistema ocupacional, cuya ubicación en el subempleo precario impide la movilidad ascendente de estos trabajadores, como del espacio de ciudadanía, cuyo caso extremo lo constituye esa población en continua rotación espacial, se ha plasmado un programa de ordenación del territorio, en el que el inmigrante es continuamente relegado al gueto.

La presión de las comunidades locales, la ausencia de posibilidades de alquilar vivienda, el rechazo de los propietarios de bares que impiden la entrada de "moros», la presión de la policía, etc., delimitan progresivamente la topología donde pueden asentarse los inmigrantes. Se ven empujados a vivir en los campos donde trabajan, fuera de las localidades, dispersos por las fincas, habitando casas derruidas, antiguas naves o corrales de ganado en condiciones ruinosas, en chabolas de autoconstrucción, etc., siempre en situación de infravivienda. Esta situación impide iniciar los procesos administrativos para obtener la reunificación familiar. Y así se crea un círculo de retroalimentación de la exclusión (Pedreño, 1999b). 


\section{Trabajadores nómadas a la búsqueda de subsistencia}

En la región de Murcia hemos venido observando un fenómeno de nomadismo laboral, al que conviene prestar atención para entender las biografías laborales que se están acuñando. Numerosos mercados de trabajo locales están generando un excedente de población creciente, que se ve empujado a incorporarse a estrategias de movilidad territorial, de itinerancia entre el lugar de residencia (el pueblo como espacio de reproducción) y el lugar de trabajo (como espacio de producción). Estos procesos de nomadismo laboral son cuantitativamente significativos en la construcción, la agricultura, la industria agroalimentaria, la hostelería, etc., y suministran una fuerza de trabajo móvil y eventual $^{7}$.

Su principal característica es su polivalencia o itinerancia entre ocupaciones (siempre en mercados de trabajo cuyas reglas de juego sean conocidas: jornalerismo, ajuste verbal, redes informales de reclutamiento, etc.). Son figuras obreras híbridas o mixtas, que oscilan entre una pluralidad de subempleos muy diversificada. Su cultura de trabajo no se define tanto en función de una identidad profesional, sino más bien dentro de una lógica social de supervivencia. Auténticos estrategas del «buscarse la vida», también tendrán una notable disponibilidad para largos desplazamientos territoriales a la búsqueda de empleo.

Para estos trabajadores, dotados de una gran capacidad de movilidad territorial, sus desplazamientos diarios mediante coches particulares o en «furgonetas» de empresas contratistas, se convierten en la forma normal de "estar» en el mercado de trabajo. Ante la ausencia de trabajo en la localidad, o la opción de liberarse de unas condiciones locales de empleo sentidas como negativas o indignas, se ven empujados a una movilidad espacial hacia «afuera».

Los trayectos en autobús (o en furgoneta o coches particulares) hasta el lugar de trabajo suponen para los obreros invertir a menudo dos o tres horas diarias de desplazamiento. Este tiempo no es remunerado por las empresas donde estos «obreros móviles» trabajan. Sin embargo, el tiempo invertido en desplazamiento es, en realidad, tiempo de trabajo no remunerado, ya que alarga considerablemente la jornada laboral.

La permanente movilidad espacial imprime un determinado ritmo a la vida social de los agentes participantes de la misma. Podríamos afirmar que constituye una pauperización de la vida cotidiana de los «obreros nómadas», en cuanto

7. Se carecen aún de monografías específicas sobre estos fenómenos de movilidad. En Oliva (1995) se analiza detenidamente el caso de los más de 15.000 trabajadores de la construcción de Castilla-La Mancha que se desplazan diariamente desde sus pueblos de residencia al mercado de trabajo del área metropolitana de Madrid. En la región de Murcia también existe esta movilidad de los trabajadores de la construcción hacia Madrid y hacia las localidades costeras del Mediterráneo, pero desconocemos la naturaleza cuantitativa y cualitativa del flujo. Los procesos de movilidad de trabajadores de la hostelería también son muy intensos a lo largo de la orilla mediterránea, pero igualmente desconocemos la existencia de estudios sociológicos específicos. Puede consultarse Pedreño (1998 y 2000a), donde se realizan estudios cualitativos de las redes de trabajadores agrícolas que recorren toda la región y, con menos profundidad, también sobre los obreros de la construcción. 
que su uso diario del tiempo queda absolutamente subsumido en la dinámica del nomadismo laboral. Las horas del día quedan distribuidas en función de la condición móvil de esos obreros: 1) la jornada laboral propiamente dicha, más el tiempo de la comida en el lugar de trabajo, 2) las horas de viaje y 3) en el caso de las mujeres, el trabajo doméstico de dejar comida preparada para los hijos, hacer las labores del hogar, hacer la comida para llevar al día siguiente al lugar de trabajo, etc.

\section{Conclusiones: la producción de biografías sociales precarizadas}

Los indicios presentados sobre la progresiva instauración de una importante bolsa de población obrera eventual en la región de Murcia ilustran un proceso de deterioro de las condiciones de vida de muchas personas, al incorporar a su experiencia una esfera cada vez más grande de incertidumbre y riesgo. Al igual que Wright Mills (1957: 110) explicó la formación del estrato de "los muy ricos» constatando la existencia de un «ciclo de acumulación de ventajas» que combinaba disposición psicológica y oportunidades objetivas, por nuestra parte podemos señalar que la población obrera de la sociedad del riesgo está progresivamente incorporándose a un proceso paralelo, pero a la inversa, de acumulación de desventajas, y que proponemos denominar mecanismo de retroalimentación de la precariedad.

El funcionamiento de este mecanismo unifica un nivel objetivo y un nivel subjetivo en interacción recíproca. En el nivel objetivo es donde institucionalmente se ha producido un proceso de desregulación del mercado de trabajo que lo ha fragmentado y precarizado. En el nivel subjetivo, las personas que subsisten en ese mercado se ven envueltas en una espiral de precariedad que les hace desarrollar una falta de expectativas y una ausencia de confianza en que las cosas podían ser de otra forma, que retroalimenta la precariedad del entorno laboral y, en definitiva, las aleja de la ciudadanía social plena. Este aspecto fue tratado por Marshall de forma muy insuficiente en su teoría de la ciudadanía, y cuestiona en profundidad su concepción del ciudadano como «una persona libre e independiente protegida por derechos». Además, se ha constatado que las desigualdades de estatus y género tienen un peso significativo. En términos weberianos podemos afirmar que el género y la etnia no son fenómenos económicamente condicionados, en cuanto que se forjan en la estructura social, pero sí fenómenos económicamente relevantes, en cuanto que dichas desigualdades están siendo utilizadas en los modos de organización de la producción.

La producción de riesgo en nuestras sociedades avanzadas está teniendo un efecto de distribución muy desigual, lo que conduce a un retroceso de la esfera de ciudadanía social, especialmente en aquellos territorios donde la vulnerabilidad se sitúa como un rasgo central de su estructura social y ocupacional. En sentido duro, puede afirmarse que la estrategia de valorización económica fundamentada en el «trabajo barato» presente hoy de forma dominante en la región de Murcia produce subjetividades sociales precarizadas. Estas sub- 
jetividades conforman lo que Beck (1998b: 210) denomina «peligros biográficos», al referirse a "esa situación en la cual una cantidad cada vez mayor de hombres se encuentra arrastrada por relaciones que no es capaz de entender, dominar o ignorar por sus propios medios o capacidades». Son vidas condicionadas por prácticas sociolaborales que le expropian progresivamente de su capacidad reflexiva.

El resultado es un territorio de vulnerabilidad social con tendencia a su reproducción ampliada. La eclosión final de esta lógica es la configuración de una «región vulnerable». Allá donde se da una subsunción real del territorio social a estas estrategias económicas de valorización sostenidas sobre el trabajo barato, asistimos a un deterioro de la ciudadanía, expresado en la debilidad de la participación social en asuntos colectivos, en una baja percepción de los procesos educativos formales, en familias desestructuradas, etc. Habría que investigar más para comprobar si la baja tasa de estudios existente en estas «regiones vulnerables», el alto fracaso escolar, etc. no son sino efectos sobre los habitus de las posiciones sociales más afectadas por ese proceso de subsunción.

Igualmente, estos territorios sociales precarizados presentan un cúmulo de desventajas para su inserción en esa nueva "civilización única» que se anuncia bajo el término de "sociedad de la información». Si la formación permanente es la regla del juego de esa estructura social informacional, es de temer que, a causa de las razones que venimos esgrimiendo, las regiones vulnerables queden relegadas. Una vez el círculo de acumulación de precariedad arraiga en un territorio, los procesos de aprendizaje quedan debilitados. Ello unido a que los sistemas productivos no incentivan la formación, sus estrategias de organización del trabajo no son cualificantes, buscan de forma permanente la desvalorización de la mano de obra, etc. De esta forma, el peligro biográfico también irrumpe con fuerza en el terreno de la formación, generando una débil propensión hacia el aprendizaje profesional o el reciclaje ${ }^{8}$.

Las investigaciones de Enrico Pugliese, Costis Hadjimichalis y Enzo Mingione ${ }^{9}$, sobre las regiones del sur de Europa, han caracterizado una forma de estructuración social específica dentro de las mismas, el «sur del Sur», es decir, espacios regionales que dentro del sur de Europa compartieron en el periodo fordista su condición de espacio periférico de la sociedad industrial. Lo que se observa con el advenimiento de la crisis de la sociedad industrial, y

8. Numerosos estudios coinciden en resaltar este efecto: «Cuando los individuos se encuentran en condiciones de inseguridad existencial, de falta de perspectivas y de confianza en relación con la mejora de la situación profesional, no están reunidas las condiciones mínimas para poder responsabilizarlos por la renovación de sus facultades. Esta responsabilidad sólo es posible cuando el individuo se encuentra, en su situación de trabajo y en su vida en general, con una posición en el mercado de trabajo interno y externo que le confiere confianza, que le estimula a pensar en proyectos de futuro y, al mismo tiempo, le confiere la posibilidad, el interés y la capacidad de autoaprendizaje» (Kovács, 1998: 18).

9. Véase Hadjimichalis, C. y Vaiou, D. (1998), Mingione, E. (1993) y (1988), y Mingione, E. y Pugliese, E. (1995). 
con el posterior proceso constitutivo de la sociedad de la información, es que las regiones del «sur del Sur» persisten en su condición de «regiones frágiles» dentro de la Unión Europea.

\section{Bibliografía}

Alonso, L.E.; CONDE, F. (1996). «Las paradojas de la globalización, la crisis del estado de bienestar nacional y las regiones vulnerables». Revista de Estudios Regionales, 44, enero-abril.

BAGNASCO, A. (1997). «Italia: cambio social en tiempos de cambio político». Sociología del Trabajo, 31, nueva época, otoño.

BECK, U. (1997). The Reinvention of Politics: Rethinking Modernity in the Global Social Order. Cambridge: Polity.

- (1998a). La sociedad del riesgo. Barcelona: Paidós.

- (1998b). ¿Qué es la globalización? Barcelona: Paidós.

BeCK, W.; MAESEN, L.; WALKeR, A. (eds.) (1997). The Social Quality of Europe. Kluwer: Law International.

BONNANO, A. (1994). «Globalización del sector agrícola y alimentario: crisis de convergencia contradictoria». En BONNANO, A. (ed.) (1994). La globalización del sector agroalimentario. Madrid: MAPA.

BotTomore, T. (1998). «Ciudadanía y clase social, cuarenta años después». En MARShall, T.H. (1998/e.o. 1950). Ciudadanía y clase social. Madrid: Alianza Editorial.

Boyer, R. (1986). La flexibilidad del trabajo en Europa. Ministerio de Trabajo y de Seguridad Social.

Bourdieu, P. (1999a). Meditaciones Pascalianas. Anagrama.

- (1999b). Contrafuegos: reflexiones para servir a la resistencia contra la invasión neoliberal. Barcelona: Anagrama.

CAMPILlO, A. (1985). Adios al progreso. Anagrama.

CASTEL, R. (1995). «De la exclusión como estado a la vulnerabilidad como proceso». Archipiélago, 21, verano.

COMISIÓN EUROPEA (1995). El libro verde de la innovación. Bruselas.

Ellison, N. (1997). «Towards a New Social Politics: Citizenship and Reflexivity in Late Modernity». Sociology, vol. 31, 4, noviembre.

ETXEZARRETA, M. (1994). «Trabajo y agricultura: los cambios del sistema de trabajo en una agricultura en transformación». Agricultura y Sociedad, 54.

Giddens, A. (1996). «T.H. Marshall, the State and Democracy». En Bulmer, M.; ReES, A.M. (ed.). Citizenship Today. The Contemporary Relevance of T.H. Marshall. Londres: UCL Press.

Hadjimichalis, C.; VaIOU, D. (1998). «Informalisation in Along Global Commodity Chains Some Evident From Southern Europe». En YBARRA, J.-A. (ed.) (1998). Economía sumergida: el estado de la cuestión en España. Murcia: UGT.

Hindess, B. (1993). "Citizenship in the Modern West». En Turner, B.S. (ed.). Citizenship and Social Theory. Londres: Sage Publications.

KovÁCS, I. (1998): «Trabajo, cualificaciones y aprendizaje a lo largo de la vida. Ilusiones y problemas de la sociedad de la información». Sociología del Trabajo, nueva época, 34 , otoño.

MARSHALL, T.H. (1998/e.o. 1950). Ciudadanía y clase social. Madrid: Alianza Editorial. 
Mingione, E. (1988). "Work and Informal Activities in Urban Southern Italy». En PAHL, R.E. (ed.). On Work: Historical Comparative and Theoretical Approaches. Oxford: Basil Blackwell.

- (1993). Las sociedades fragmentadas. Madrid: Ministerio de Trabajo y de Seguridad Social.

Mingione , E.; Pugliese, E. (1995). «Modelli Occupazionali e Disoccupazione Giovanile di Massa nel Mezzogiorno». Sociologia del Lavoro, 59-60.

MONREAL, J. (1986). «Espacios periféricos y población». Monográfico sobre Desigualdad $y$ dependencia. La periferización del Mediterráneo occidental (siglos XII-XIX). Áreas. Murcia.

- (1999). «El futuro del medio ambiente: entre la globalización y la participación local». En Libro homenaje al profesor doctor Joaquin Moreno Clavel. Universidad de Murcia.

MONREAL, J. y otros (1991). "Problemas y condiciones de vida de la población en la región de Murcia». Áreas. Murcia.

OLIVA, J. (1995). Mercados de trabajo y reestructuración rural. Una aproximación al caso castellano-Mmanchego. Madrid: MAPA.

ORTí, A. (1992). «Para una teoría de la sociedad de las clases medias funcionales de los 80». Documentación Social, 88.

PAHL, R.E. (ed.) (1988). On Work: Historical Comparative and Theoretical Approaches. Oxford: Basil Blackwell.

Pedreño, A. (1998). «Economía flexible y ruralidad: el caso de la vega media del río Segura en la región de Murcia». En YBARRA, J.-A. (ed.) (1998). Economía sumergida: el estado de la cuestión en España. Murcia: UGT.

- (1999a). «Taylor y Ford en los campos. Trabajo, género y etnia en el cambio organizacional y tecnológico de la agricultura murciana». Sociología del Trabajo, nueva época, 35. Madrid.

- (1999b). «Construyendo la huerta de Europa: trabajadores sin ciudadanía y nómadas permanentes en la agricultura murciana». Migraciones, 5. Madrid.

- (2000a). Del jornalero agrícola al obrero de las factorías vegetales: estrategias familiares y nomadismo laboral en la ruralidad murciana. Madrid: Servicio de Publicaciones del Ministerio de Agricultura, Pesca y Alimentación.

- (2000b). Ruralidad Globalizada. Sociología de los territorios de las factorías vegetales. Murcia: Diego Marín Editores.

PRIES, L. (1991). «El cambio industrial en las sociedades modernas como sociedades de riesgo». Sociología del Trabajo, nueva época, 12, primavera.

Secretaría General de Economía y Planificación y CIS (1986). Condiciones de vida y de trabajo en España. Madrid: Ministerio de Economía-CIS.

TaYlor-Gooby, P. (1986). "Collectivism in Six Countries». En Jowell, R.; WitherSPOON, S. (dirs.). International Social Attitudes. Aldershot: Gower.

ThOmas, R. (1985). Citizenship, Gender and Work: Social Organization of Industrial Agriculture. University of California Press.

Turner, B.S. (ed.) (1993). Citizenship and Social Theory. Londres: Sage Publications. Valera, J.; Álvarez-Uría, F. (1989). Sujetos frágiles. México: Fondo de Cultura Económica.

Wallerstein, I. (1997). «Social Science and the Quest for a Just Society». American Journal of Sociology, volumen 102, 5, marzo.

Wright Mills, C. (1957). Las elites de poder. México: Fondo de Cultura Económica. 